\title{
Formulation, Preparation and Evaluation of an Intravenous Emulsion Containing Brucea Javanica Oil and Coix Seed Oil for Anti-tumor Application
}

\author{
Ye-Ling Yu, Yan Lu, Xing Tang, ${ }^{*}$ and Fu-De CuI \\ Department of Pharmaceutics, Shenyang Pharmaceutical University; Wen Hua Road, No. 103, Shenyang 110016, People's \\ Republic of China. Received September 20, 2007; accepted January 11, 2008; published online January 21, 2008
}

\begin{abstract}
The purpose of this study was to prepare and evaluate the intravenous emulsion (BCOE) containing Brucea javanica oil (BJO) and Coix seed oil (CSO), which is used in anti-tumor treatment. The formulation and preparation of $\mathrm{BCOE}$ were systematically investigated. High-pressure homogenization, particle size distribution, $\zeta$-potential and HPLC were carried out. The pharmacokinetics of the main component, oleic acid, and anti-tumor activity studies about the tumor growth inhibitory ratios (TGIR) and the mortality experiments were also employed to evaluate BCOE in vivo compared with BJO emulsion (BJOE) and CSO emulsion (CSOE) using $\mathrm{S}_{180}$ sarcoma-bearing mice. The final BCOE formulation was $10 \%(w / v)$ oils with BJO and CSO $3: 1,0.6 \%$ (w/v) Lipid E 80, 0.3\% (w/v) Pluronic F-68 (F-68), 0.1\% (w/v) sodium oleate and $2.5 \%$ (w/v) glycerin in water. The preparation conditions involved $70^{\circ} \mathrm{C}$ for preparing the crude emulsion, 6 cycles for high-pressure homogenization at 500 bar, $\mathrm{pH}$ value was adjusted to 8.5 after high-pressure homogenization and $115^{\circ} \mathrm{C}$ for 30 min in a rotating water bath for sterilization. The pharmacokinetics parameters showed the combination of BJO and CSO may not influence the elimination of $B C O E$ and have no significant difference between BCOE and BJOE or CSOE. The data of TGIR and mortality indicated that BCOE could increase the anti-tumor activity of CSOE and reduced the toxicity of BJOE. The mortality study (BCOE 0, BJOE 63.3\%, CSOE 13.3\%) showed that BCOE greatly reduced the toxicity of BJOE and CSOE. Therefore, the development and application of BCOE will make an important contribution to anti-tumor therapy.
\end{abstract}

Key words formulation; preparation; pharmacokinetics; emulsion; Brucea javanica oil; Coix seed oil

Fat emulsion is a special drug delivery system, which is employed to supply calories to patients or as a vehicle for lipid soluble drugs. Since the development of Intralipid ${ }^{\circledR}$ as an intravenous nutrition emulsion in 1962, fat emulsions loaded drugs have also been studied in detail in the last 40 years for their inherent advantages such as sustained release and targeting applications in the endothelial system and mononuclear phagocyte system. In addition, intravenous emulsions are now widely used in anticancer therapy because of the advantages described above. ${ }^{1,2)}$

Malignant tumors are one of the most serious conditions posing a great threat to patients' lives. There are many commercially available anti-tumor drugs for their treatment. Brucea javanica oil emulsion (BJOE) and Coix seed oil emulsion (CSOE) have already been developed for antitumor clinical applications. ${ }^{3)}$ BJO is extracted from the nucleoli of Brucea javanica (L.) Merr. (Simaroubaceae) which is widely distributed from southeast Asia to northern Australia. ${ }^{4)} \mathrm{CSO}$ is obtained from the seed of Coix lacryma-jobi L. var. mayuen STAPF by supercritical fluid extraction with $\mathrm{CO}_{2}\left(\mathrm{SFECO}_{2}\right)$. Both oleic acid and linoleic acid are the active components of $\mathrm{BJO}$ and $\mathrm{CSO}$, and they play an important role in anti-tumor treatment. ${ }^{5,6)}$ BJOE and CSOE are used to treat primary malignant tumors, including lung cancer, liver cancer, gastric cancer, and breast cancer, because of their wide anti-tumor spectra and good therapeutic effects. They enhance immunocompetence and the effect of natural killer cells on tumor cells. As for CSOE, it has no adverse effects on heart, liver, kidney and hemopoietic system, which encourages improved patient compliance. Furthermore, the mechanism of anti-tumor activity had been thoroughly investigated for both oils. The anti-tumor effect of BJO involves inhibiting the synthesis of DNA, RNA and protein in tumor cells, inducing tumor apoptosis and reversing multidrug resistance, while the anti-tumor effect of CSO involves holding up caryocinesia in tumor cells, inducing tumor apoptosis, inhibiting the formation of new tumor vessels, influencing oncogene expression, modifying cytokine levels and reversing multidrug resistance. ${ }^{7,8)}$

In this paper, the formulation and preparation of an intravenous emulsion involving combined $\mathrm{BJO}$ and $\mathrm{CSO}$ were systematically investigated. The combined use of BJO and CSO to prepare Brucea javanica oil and Coix seed oil emulsion (BCOE) showed the better therapeutic effect. The most valuable aspect of BCOE is that the use of a combined prescription of the two oils improves the anti-tumor activity of CSOE and increases the tolerated dose of BJO while clearly reducing its toxicity. Furthermore, pharmacokinetics and anti-tumor activity studies were also carried out. All the results obtained strongly supported the development of BCOE because of its improved anticancer effect and lower toxicity.

\section{MATERIALS AND METHODS}

Materials Brucea javanica oil was supplied by Yingkou Newport Medicinal Herbs Refinery (Yingkou, China); Coix seed oil was obtained from Guangzhou Masson Pharmaceutical Ltd. (Guangzhou, China); Lipoid E 80 was purchased from Lipoid KG (Ludwigshafen, Germany) and Plutonic F68 (F-68) was obtained from BASF (German). S 180 sarcoma strain was purchased from Immunization Laboratory of China Medical University (Shenyang, China). All other chemicals and reagents were of analytical or chromatographic grade.

Purification of Coix Seed Oil and Brucea Javanica Oil To achieve the standard of oils for injection, Coix seed oil 
and Brucea javanica oil were purified as follows. Coix seed oil was placed in a $250 \mathrm{ml}$ conical flask and heated on a water bath, then $5-10 \% \mathrm{NaOH}$ alcoholic solution was added dropwise to neutralize the free fatty acid until the dihydroxyphthalophenone indicator solution turned red. Then, the oil was separated and washed with $70^{\circ} \mathrm{C}$ water for injection repeatedly to remove saponified soap and excess alkali until the oil displayed a yellow color. The oil was isolated, $0.5 \%$ activated carbon was added followed by heating to $80^{\circ} \mathrm{C}$ and agitating for $15 \mathrm{~min}$, then filtering the hot solution. The oil was separated again and deodorized by vapor for injection and then the oil was isolated. Anhydrous calcium chloride for injection was added as a drying agent and then the oil was allowed to stand for $12-24 \mathrm{~h}$ and finally passed through a filter to obtain purified Coix seed oil. ${ }^{9)}$ Brucea javanica oil was purified in the same way.

Emulsion Preparation BJO and CSO of $10 \%(w / v)$ at a ratio of $3: 1$ together with Lipid E 80 were dissolved as the oil phase at $70^{\circ} \mathrm{C}$; F-68, glycerin and sodium oleate were used as the aqueous phase and heated in a water bath at $70^{\circ} \mathrm{C}$ until completely dissolved. In addition, the water phase was rapidly added drop-wise to the oil phase followed by high speed shear mixing (ULTRA RURRAX ${ }^{\circledR}$ IKA $^{\circledR}$ T18 basic, Germany) at $20000 \mathrm{rpm}$ for $8 \mathrm{~min}$. The coarse emulsion was made up to $100 \mathrm{ml}$ with the water for injection then subjected to high-pressure homogenization (Niro Soavi NS10012k homogenization, Via M. da Erba, 29/A-43100 PARMA, Italy) at 500 bar for 6 cycles to obtain final emulsion, and the whole process was carried out at $40^{\circ} \mathrm{C}$ cooled by an ice-water bath. Then, the final emulsion was adjusted to $\mathrm{pH} 8.5$ with 0.1 $\mathrm{mol} / \mathrm{l} \mathrm{NaOH}$ or $\mathrm{HCl}$. Finally, nitrogen was passed through the emulsion before it was sealed in vials and sterilized in a rotating water vapor bath at $115^{\circ} \mathrm{C}$ for $30 \mathrm{~min}$. BJOE and CSOE were also prepared as the procedure and formulation described above with the oil phase of BJO or CSO $10 \%$ $(\mathrm{w} / \mathrm{v})$, respectively.

Sterilization Procedures for Emulsions Three kinds of autoclaving methods of $121^{\circ} \mathrm{C}$ for $15 \mathrm{~min}, 105^{\circ} \mathrm{C}$ for $45 \mathrm{~min}$ and $115^{\circ} \mathrm{C}$ for $30 \mathrm{~min}$ were selected for the investigation of sterilization procedures of BCOE.

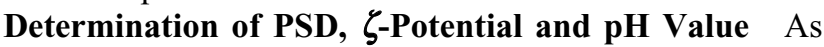
the most important parameters to investigate the physical stability of the emulsion, particle size diameter (PSD), $\zeta$-potential and $\mathrm{pH}$ value were determined. The particle size of BCOE was measured by the photon correlation spectroscopy (PCS) technique using a Nicomp ${ }^{\mathrm{TM}} 380$ Particle Sizing system (Santa Barbara, U.S.A.). The system covered a range of approximately $5 \mathrm{~nm}$ to $3 \mu \mathrm{m}$ and has, therefore, been extensively used for particle size determination of intravenous emulsions. The sample was diluted 1:5000 with double distilled water which was passed through a $0.22 \mu \mathrm{m}$ membrane immediately before measurement at $25^{\circ} \mathrm{C}$. A Nicomp ${ }^{\mathrm{TM}} 380$ system was also used to determine the $\zeta$-potential of the emulsion and the electrophoretic light scattering (ELS) technique was used. The emulsion sample was diluted $1: 50$ with the double distilled water adjusted to the same $\mathrm{pH}$ as the emulsion using $0.01 \mathrm{~mol} / 1 \mathrm{HCl}$ or $\mathrm{NaOH}$. The determination was carried out at $25^{\circ} \mathrm{C}$ and the $\mathrm{pH}$ of the bulk emulsion was measured using a pH-meter (Shanghai Precision Science Instrument Ltd., China) with a glass microelectrode at room temperature $\left(25 \pm 2{ }^{\circ} \mathrm{C}\right)$.
Pharmacokinetics Study of BCOE Pharmacokinetics experiment: Wistar rats weighing $250 \pm 20 \mathrm{~g}$ were divided into 3 groups, each with 6 rats, by randomization, and these represented BJOE, CSOE and BCOE groups. The dose administered to each group was $2 \mathrm{ml} / \mathrm{kg}$ by injection via the femoral vein. Rats were anesthetized with ether when sampling and $0.2 \mathrm{ml}$ blood samples were obtained by retro-orbital puncture at $0,2,5,15,30,60$ and $120 \mathrm{~min}$. The experimental procedures complied with the University Animal Ethics Committee Guidelines.

Extraction of Plasma and Pre-column Derivatization of Oleic Acid from Rat Plasma before UV-HPLC Determination (a) Extraction of Oleic Acid from Plasma: The blood samples obtained were immediately centrifuged at $4000 \mathrm{rpm}$ for $10 \mathrm{~min}$, and plasma was obtained from the supernatant. Then, $200 \mu \mathrm{l}$ distilled water was added to $50 \mu \mathrm{l}$ plasma, and vortexed for $2 \mathrm{~min}$. A $50 \mu \mathrm{l}$ sample of the upper solution was put into a $10 \mathrm{ml}$ conical tube, $150 \mu \mathrm{l}$ distilled water was added following by $20 \mu \mathrm{l}$ of an internal standard solution of heptadecanoic acid, and a $400 \mu \mathrm{l}$ solution of isopropanolether-phosphoric acid $(2 \mathrm{M})(40 / 10 / 1 \mathrm{v} / \mathrm{v})$. After vortexed for $5 \mathrm{~min}, 200 \mu \mathrm{l}$ distilled water was added following by $1 \mathrm{ml}$ ether and then vortexed for another $5 \mathrm{~min}$. After centrifuged at $4000 \mathrm{rpm}$ for $5 \mathrm{~min}$, the supernatant was removed. ${ }^{10}$ )

(b) Pre-column Derivatization of Oleic Acid in Rat Plasma before UV-HPLC Determination ${ }^{11-14)}$ : Because oleic acid is only of UV end absorption, pre-column derivatization was selected to obtain high sensitivity of it by UV-HPLC determination. The sample obtained by the above procedure was evaporated to dryness under nitrogen. $0.8 \mathrm{ml} 0.5 \mathrm{~mol} / 1 \mathrm{KOH}-$ methanol solution was added to the sample, which was then saponified in a $60^{\circ} \mathrm{C}$ water bath for 30 min until the oil was sufficiently dissolved following by evaporation to dryness under nitrogen. This process was followed by addition of $1 \mathrm{ml}$ water for injection and drops of $6 \mathrm{M} \mathrm{HCl} \mathrm{until} \mathrm{the} \mathrm{dihy-}$ droxyphthalophenone indicator color faded. The sample was then extracted twice with $1.6 \mathrm{ml}$ ether and each time the sample was vortexed for $2 \mathrm{~min}$. Using dihydroxyphthalophenone as the indicator, the mixture was neutralized with $0.05 \mathrm{~mol} / 1$ $\mathrm{KOH}-$ ethanol solution and then was evaporated to dryness under nitrogen. Then, $60 \mu \mathrm{l} 2,4^{\prime}$-diphenacyl bromide was added, followed by $60 \mu \mathrm{l} 18$-corona-6-ether solution and $600 \mu \mathrm{l}$ acetonitrile, the sample was vortexed for $2 \mathrm{~min}$ and mixed by ultrasound for another $1 \mathrm{~min}$ to sufficiently mix. Then, the mixture was heated at $80^{\circ} \mathrm{C}$ in a water bath for $40 \mathrm{~min}$ with application of ultrasound for $30 \mathrm{~s}$ every 10 min. ${ }^{15,16)}$ Finally, the sample was allowed to cool, then was centrifuged, and the supernatant was used for HPLC determination.

Determination of Oleic Acid in Plasma by HPLC An HPLC analytical method was used for drug analysis involving a Kromasil $\mathrm{C}_{8}$ column $(5 \mu \mathrm{m}, 4 \mathrm{~mm} \times 250 \mathrm{~mm}$, KYA TECH Corporation). The mobile phase consisted of acetonitrile-distilled water $(75: 25)$ at a flow rate of $1.3 \mathrm{ml} / \mathrm{min}$; the UV detection wavelength was $254 \mathrm{~nm}$, the column temperature was ambient and the injected volume was $20 \mu \mathrm{l}$. Since oleic acid was the main component of BCOE and was present at a relatively low concentration in blank rat, and also the most important anti-tumor component of $\mathrm{BCOE}$, it was taken as the essential component for determination. In addition, at each time point, the oleic acid concentration measured was 
corrected by the oleic acid concentration measured at 0 min time point.

Anti-tumor Activity Studies of BCOE (a) The Determination of the tumor growth inhibitory ratio (TGIR): Innoculation: $\mathrm{S}_{180}$ sarcoma-bearing ascitic mice of which the tumor growth is productive were selected for inoculation. The $\mathrm{S}_{180}$ sarcoma-bearing ascitic mice were put to death by cervical vertebrae luxation, and then fixed to operation plank, disinfected by Iodophors and alcohol in turns. In asepsis condition, the mice were taken out ascitic fluid. The ascitic fluid was diluted with isotonic $\mathrm{Na}$ chloride to $1 \times 10^{6}-10^{7} \mathrm{cell} / \mathrm{ml}$ to prepare neoplastic cell suspension, and the suspension was inoculated to the armpit of the normal Kunming-species mice by $0.2 \mathrm{ml}$ each one. $24 \mathrm{~h}$ after inoculation, the mice could be used for the following experiments.

Anti-tumor activity was evaluated in $\mathrm{S}_{180}$ sarcoma-bearing mice by measuring TGIR. The animals were divided into 8 groups of 12 mice by randomization according to the body weight $24 \mathrm{~h}$ after inoculation with $\mathrm{S}_{180}$ sarcoma. All the animals treated as described bellow immediately after dividing them into groups. The 8 groups were separately administered physiological saline (negative control group, via tail vein), blank lipid emulsion $(10 \mathrm{ml} / \mathrm{kg} / \mathrm{d}$, via tail vein) which was stabilized with the same emulsifiers used to stabilize the BCOE, BJOE $(10 \mathrm{ml} / \mathrm{kg} / \mathrm{d}$, via tail vein), CSOE $(10 \mathrm{ml} / \mathrm{kg} / \mathrm{d}$, via tail vein), and low, medium and high doses of $\mathrm{BCOE}$ $(5 \mathrm{ml} / \mathrm{kg} / \mathrm{d}, 10 \mathrm{ml} / \mathrm{kg} / \mathrm{d}, 20 \mathrm{ml} / \mathrm{kg} / \mathrm{d}$; via tail vein) and $\mathrm{BCOE}$ $(10 \mathrm{ml} / \mathrm{kg} / \mathrm{d}$, p.o. $)$. The doses were administered once daily for $7 \mathrm{~d}$. To determine the TGIR, the tumors were surgically removed, weighed and visually examined $24 \mathrm{~h}$ after the last administration. The experimental procedures complied with the University Animal Ethics Committee Guidelines. The experiment was performed in triplicate. The TGIR was obtained as the following equation:

$\operatorname{TGIR}(\%)=\{[\mathrm{ATW}(\mathrm{CG})-\mathrm{ATW}(\mathrm{MAT})] / \mathrm{ATW}(\mathrm{CG})\} \times 100 \%$

ATW (average tumor weight), MAT (medication administration team), CG (control group).

(b) The Determination of Mortality: The reduction in toxicity of BCOE was evaluated with $\mathrm{S}_{180}$ sarcoma-bearing mice by determining the mortality compared with BJOE and CSOE. The animals were randomly divided into 4 groups of 10 mice according to body weight $24 \mathrm{~h}$ after inoculation with $\mathrm{S}_{180}$ sarcoma. The 4 groups were separately given physiological saline (cancer-bearing control group, via tail vein), BJOE (via tail vein), CSOE (via tail vein), BCOE (via tail vein). The administered volume was $20 \mathrm{ml} / \mathrm{kg} / \mathrm{d}$ for each group. The administration was once daily for $7 \mathrm{~d}$. Then, $24 \mathrm{~h}$ after the last administration, the number of dead animals was used to calculate the mortality, including the number of animals that died during the whole administration period. The experimental procedures complied with the University Animal Ethics Committee Guidelines.

percentage mortality $(\%)=($ animal number of death $/ 10) \times 100 \%$

Statistical Analysis The plasma concentration-time curve profiles of oleic acid and the pharmacokinetic parameters between BCOE and BJOE or CSOE were analyzed statistically using an independent samples-test. Statistical significance was set at $p<0.05$ in all cases. All the experimental data were analyzed using SPSS statistical software. The pharmacokinetic program, 3p87, designed by the Mathematics Pharmacological Committee, Chinese Academy of Pharmacology, which used the most appropriate pharmacokinetic model to describe the experimental data, was employed to analyze the plasma concentration-time curve data. The model was chosen based on the residual sum of squares and the minimum Akaike's information criterion (AIC) value. The area under the concentration versus time $\left(A U C_{0 \rightarrow t}\right)$ curve from zero to the last time point, the area under the cross product of time and plasma concentration-time curve $\left(A U M C_{0 \rightarrow t}\right)$, mean residence time $(M R T)$, clearance $(C l)$, apparent volume of distribution $(V)$, half-life $\left(t_{1 / 2}\right)$ and the elimination rate constant $\left(k_{\mathrm{e}}\right)$ of the drug were obtained using statistical moment theory. ${ }^{17}$

\section{RESULTS}

Formulation Investigation (a) The Ratio Adjustment of Lipid E 80 and F-68: To investigate the ratio of Lipid E 80 and F-68, the total concentration of emulsifiers consisting of Lipid E 80 and F-68 was kept constant (1.5\%), but their ratios were changed. Table 1 shows seven formulations with different ratios of Lipid E 80 and F-68. In addition, the amount of sodium oleate in each formulation was constant at $0.1 \% \mathrm{w} / \mathrm{v}$. It can be seen from Table 1 that only with lecithin or F-68 as an emulsifier like Formulation 1 and Formulation 7, or when the ratios of Lipid E 80 and F-68 were too large or small, like $1: 4$ and $4: 1$, shown in Formulation 2 and Formulation 6 , BCOE could not be prepared in a physically stable form or the emulsion could not be prepared at all. With only a single emulsifier, the interfacial film did not have enough strength to resist the shearing process, high pressure homogenization and thermal sterilization. Moreover, in Formulation 2 , when the lecithin was arranged regularly around the water-oil interfacial film, the amount of F-68 was not sufficient to form a compact emulsifier-film to maintain the stability of the emulsion. In contrast, in Formulation 6, al-

Table 1. Appearance and Physical Characteristics of BCOE with Seven Different Ratios of Lipid E 80 and F-68 after Sterilization in a Rotating Water Vapor Bath for $30 \mathrm{~min}$ at $115^{\circ} \mathrm{C}$

\begin{tabular}{|c|c|c|c|c|c|}
\hline Formulation & Lipid E 80 (w/v\%) & F-68 (w/v\%) & E80 : F68 & Appearance & PSD (nm) \\
\hline 1 & 1.5 & 0 & - & - & - \\
\hline 2 & 1.2 & 0.3 & $4: 1$ & Emulsion breaking & - \\
\hline 3 & 1 & 0.5 & $2: 1$ & Good condition & $212.9 \pm 61.3$ \\
\hline 4 & 0.6 & 0.9 & $1: 1.5$ & Good condition & $219.3 \pm 83.6$ \\
\hline 5 & 0.5 & 1 & $1: 2$ & Good condition & $209.7 \pm 69.8$ \\
\hline 6 & 0.3 & 1.2 & $1: 4$ & Emulsion breaking & - \\
\hline 7 & 0 & 1.5 & - & - & - \\
\hline
\end{tabular}


though the amount of F-68 was sufficient, the lecithin could not uniformly disperse in the water-oil interfacial film to emulsify $10 \%(\mathrm{w} / \mathrm{v})$ oils. Hence, both ratios in Formulation 2 and 6 produced a water-oil interfacial film which was very weak and would cause leakage of the oil phase and emulsion break-up. Formulations 3 to 5 had a good physical appearance. The particle size and physical appearance are also shown in Table 1.

(b) The Total Amount of Lipid E 80 and F-68: At a ratio of $2: 1$ between Lipid E 80 and F-68, the total concentration of Lipid E 80 and F-68 was investigated at three levels $1.5 \%$, $1.2 \%, 0.9 \%$. The experiments were performed in triplicate and the results are given in Table 2 . In addition, the amount of sodium oleate in each formulation was constant at $0.1 \%$ $(\mathrm{w} / \mathrm{v})$. The Int-Wt Nicomp distribution of BCOE with $1.5 \%$ $(\mathrm{w} / \mathrm{v})$ and $0.9 \%(\mathrm{w} / \mathrm{v})$ of Lipid E 80 and F-68 (2:1) is illustrated in Figs. 1a, b. The total amount of lecithin and F-68 was finally selected as $0.9 \%(\mathrm{w} / \mathrm{v})$ with a ratio of $2: 1$.

(c) The Investigation of the Amount of Sodium Oleate: Table 3 shows the influence of different amounts of sodium oleate on the characteristics of BCOE. Without sodium oleate or with only $0.05 \%(\mathrm{w} / \mathrm{v})$ sodium oleate, the $\mathrm{BCOE}$

Table 2. Influence of the Total Amount of Emulsifiers Consisting of Lipid E 80 and F-68 on the PSD of BCOE

\begin{tabular}{cccc}
\hline \hline Emulsifier amount (\%) & 1.5 & 1.2 & 0.9 \\
\hline PSD (nm) & $228.6 \pm 85.7$ & $239.4 \pm 76.2$ & $248.1 \pm 74.9$ \\
\hline
\end{tabular}

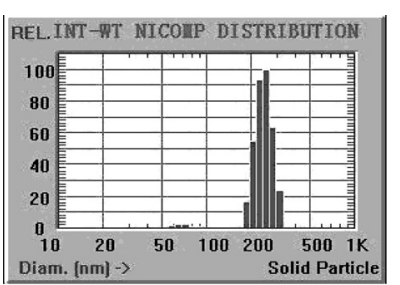

(a)

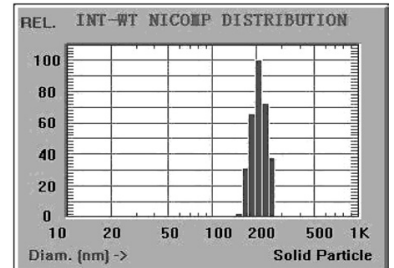

(b)
Fig. 1. The Int-Wt Nicomp Distribution of BCOE with $1.5 \%(w / v)(a)$ and $0.9 \%(\mathrm{w} / \mathrm{v})(\mathrm{b})$ of Lipid E 80 and F-68 (2:1)

(a) The amount of emulsifiers of Lipid E 80 and F-68 was $1.5 \%(\mathrm{w} / \mathrm{v})$. (b) The amount of emulsifiers of Lipid E 80 and F- 68 was $0.9 \%(\mathrm{w} / \mathrm{v})$. could not undergo thermal sterilization because the electrical repulsion would be too weak and the particles of emulsion would collide and aggregate, leading to a phase separation and a creamy appearance. Hence, $0.1 \%(\mathrm{w} / \mathrm{v})$ sodium oleate was used to prepare BCOE.

Preparation Investigation (a) Investigation of High Pressure Homogenization Cycles and Pressure: In this study, two pressure levels of 500 bar and 700 bar; 5 levels of high pressure homogenization were investigated. The data are shown in Fig. 2. The particle sizes were markedly reduced after 3 homogenization cycles. Also, from 6 to 10 cycles, the particle sizes changed only slightly. In addition, the standard deviations were larger following 10 homogenization cycles.

(b) Investigation of Sterile Methods: Three different sterile methods were used to investigate the sterile conditions and the results are shown in Table 4. The PSD and physical appearance were used to evaluate the BCOE system. It appears from Table 4 that the sterile conditions of $121^{\circ} \mathrm{C}$ for $15 \mathrm{~min}$ induce phase separation. $105^{\circ} \mathrm{C}$ for $45 \mathrm{~min}$ and $115^{\circ} \mathrm{C}$ for 30 min could both provide good physical stability with simi-

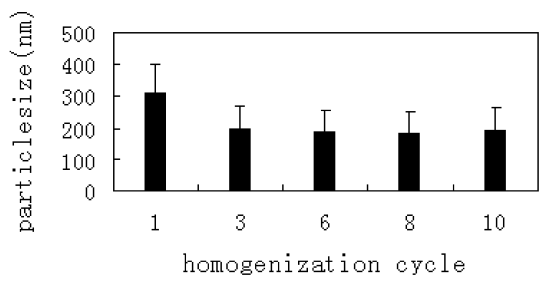

(a)

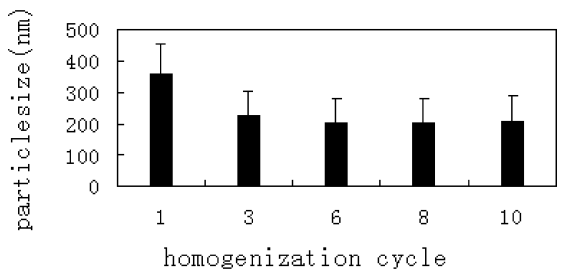

(b)

Fig. 2. Influence of High Pressure Homogenization Cycles and Pressure on the PSD of BCOE

(a) Influence of high pressure homogenization cycles on the PSD of BCOE at 700 bar. (b) Influence of high pressure homogenization cycles on the PSD of BCOE at 500 bar.

Table 3. The Effect of Different Amounts of Sodium Oleate on the Appearance and Physical Characteristics of BCOE before and after Sterilization in a Rotating Water Vapor Bath at $115^{\circ} \mathrm{C}$ for $30 \mathrm{~min}$

\begin{tabular}{|c|c|c|c|c|c|}
\hline $\begin{array}{c}\text { Amounts of sodium } \\
\text { oleate }(\%)\end{array}$ & $\begin{array}{l}\text { PSD }(\mathrm{nm}) \text { before } \\
\text { sterilization }\end{array}$ & $\begin{array}{c}\zeta \text {-Potential }(\mathrm{mV}) \\
\text { before sterilization }\end{array}$ & $\begin{array}{l}\text { Appearance after } \\
\text { sterilization }\end{array}$ & $\begin{array}{l}\text { PSD }(\mathrm{nm}) \text { after } \\
\text { sterilization }\end{array}$ & $\begin{array}{l}\zeta \text {-Potential }(\mathrm{mV}) \\
\text { after sterilization }\end{array}$ \\
\hline 0 & $244.4 \pm 56.3$ & -11.27 & Phase separation & - & - \\
\hline 0.05 & $226.7 \pm 65.2$ & -16.0 & Creaming & - & - \\
\hline 0.1 & $219.9 \pm 55.8$ & -32.0 & Homogeneous phase & $223.5 \pm 37.6$ & -36.8 \\
\hline
\end{tabular}

Table 4. Influence of Different Thermal Sterile Methods on the Physical Stability of BCOE

\begin{tabular}{|c|c|c|c|c|}
\hline & & $105^{\circ} \mathrm{C} 45 \mathrm{~min}$ & $115^{\circ} \mathrm{C} 30 \mathrm{~min}$ & $121^{\circ} \mathrm{C} 15 \mathrm{~min}$ \\
\hline Before sterilization & $\begin{array}{l}\text { PSD (nm) } \\
\text { Appearance }\end{array}$ & $\begin{array}{c}228.2 \pm 49.8 \\
\text { Homogeneous }\end{array}$ & $\begin{array}{c}228.2 \pm 49.8 \\
\text { Homogeneous }\end{array}$ & $\begin{array}{c}228.2 \pm 49.8 \\
\text { Homogeneous }\end{array}$ \\
\hline After sterilization & $\begin{array}{l}\text { PSD (nm) } \\
\text { Appearance }\end{array}$ & $\begin{array}{c}235.3 \pm 55.1 \\
\text { Homogeneous }\end{array}$ & $\begin{array}{c}232.7 \pm 58.9 \\
\text { Homogeneous }\end{array}$ & $\begin{array}{c}253.6 \pm 60.7 \\
\text { Phase separation }\end{array}$ \\
\hline
\end{tabular}


lar particle sizes and distributions. $115^{\circ} \mathrm{C}$ for $30 \mathrm{~min}$ was finally employed for sterilization.

Pharmacokinetics in Rats after Intravenous Injection of BCOE A typical plasma chromatogram is shown in Fig. 3. Figure 3 shows that the components in plasma do not interfere with their mutual determination. A comparative pharmacokinetic evaluation was carried out by measuring drug concentrations in plasma up to $2 \mathrm{~h}$ after the administration of $\mathrm{BJOE}, \mathrm{CSOE}$ and $\mathrm{BCOE}$, respectively. The main pharmacokinetic parameters were analyzed by the pharmacokinetics program 3p87. The plasma concentration-time curve is shown in Fig. 4 and the main pharmacokinetic parameters are described in Table 5. In addition, the oleic acid concentration measured was corrected by the oleic acid concentration measured at 0 min time point.

Anti-tumor Studies of BCOE The results of TGIR of different medication administration teams and determination of mortality (the dose was $20 \mathrm{ml} / \mathrm{kg} / \mathrm{d}$, administered via the tail vein) are shown in Tables 6 and 7, respectively.
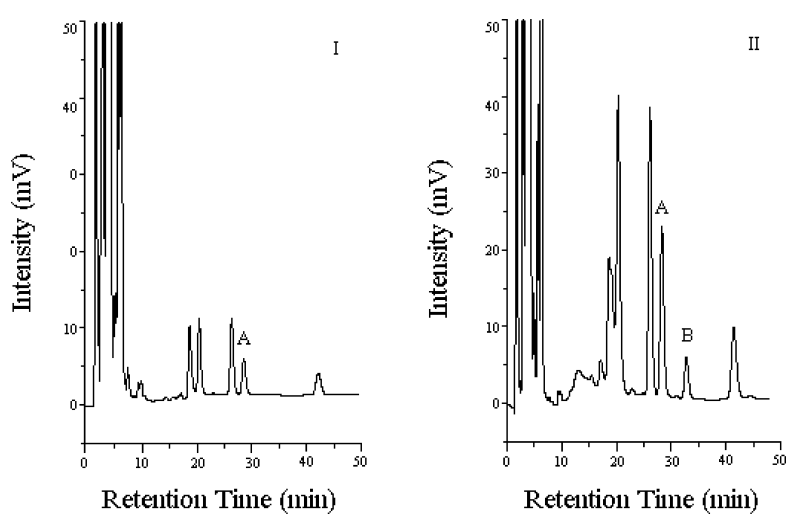

Fig. 3. Typical Chromatograms of Rat Plasma (Peak A: Oleic Acid; Peak B: Heptadecanoic Acid) for Pharmacokinetics Evaluation

I, Blank plasma sample (without internal standard); II, Plasma sample (with internal standard).

\section{DISCUSSION}

Formulation Investigation (a) The Ratio Adjustment of Lipid E 80 and F-68: Emulsification is a very important process for emulsion preparation and this plays an essential

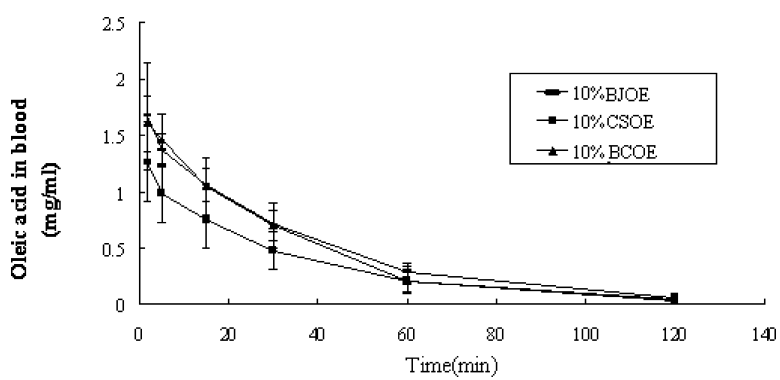

Fig. 4. The Plasma Concentration-Time Curve of Oleic Acid after Intravenous Administration of $\operatorname{BCOE}(\boldsymbol{\Delta}), \operatorname{BJOE}(\boldsymbol{-})$ and $\operatorname{CSOE}(\boldsymbol{\square})$ to Rats $(n=6)$ at the Dose of $2 \mathrm{ml} / \mathrm{kg}$

Table 5. Pharmacokinetics Parameters after Administration (i.v.) of BJOE, CSOE and BCOE

\begin{tabular}{lccc}
\hline \multicolumn{1}{c}{ Parameters } & BJOE & CSOE & BCOE \\
\hline$C_{\max }(\mathrm{mg} / \mathrm{ml})$ & $1.728 \pm 0.404$ & $1.265 \pm 0.354$ & $1.637 \pm 0.246$ \\
$k_{\mathrm{e}}\left(\mathrm{min}^{-1}\right)$ & $0.0276 \pm 0.0048$ & $0.0328 \pm 0.0096$ & $0.0346 \pm 0.0112$ \\
$t_{1 / 2}(\mathrm{~min})$ & $25.74 \pm 4.39$ & $22.84 \pm 7.22$ & $21.91 \pm 7.12$ \\
$A U C_{0 \rightarrow t}\left(\mathrm{mg} / \mathrm{ml} \cdot \mathrm{min}^{-1}\right)$ & $58.8 \pm 9.5$ & $41.9 \pm 15.0$ & $54.9 \pm 13.6$ \\
$A U C_{0 \rightarrow \infty}\left(\mathrm{mg} / \mathrm{ml} \cdot \mathrm{min}^{-1}\right)$ & $61.3 \pm 10.3$ & $43.2 \pm 15.3$ & $56.6 \pm 14.8$ \\
$A U M C_{0 \rightarrow 120}$ & $1735.8 \pm 371.8$ & $1213.7 \pm 509.7$ & $1455.5 \pm 554.7$ \\
$A U M C_{0 \rightarrow \infty}$ & $1919.1 \pm 586.3$ & $1307.9 \pm 520.1$ & $1601.5 \pm 762.1$ \\
$M R T(\mathrm{~min})$ & $30.9 \pm 4.5$ & $29.9 \pm 2.9$ & $27.2 \pm 6.8$ \\
$V(\mathrm{ml})$ & $15.1 \pm 2.1$ & $14.8 \pm 4.2$ & $14.7 \pm 2.9$ \\
$C l(\mathrm{ml} / \mathrm{min})$ & $0.494 \pm 0.068$ & $0.500 \pm 0.151$ & $0.563 \pm 0.155$
\end{tabular}

The content of oleic acid in BCOE, BJOE and CSOE was 5.6\%, 5.91\% and $4.72 \%$, respectively. The data are means \pm S.D., $n=6$. $k_{\mathrm{e}}$, elimination rate constant; $t_{1 / 2}$, half-life; $A U C_{0-t}$, area under the concentration-time curve; $A U M C_{0-t}$, area under the cross product of time and plasma concentration-time curve; $M R T$, mean residence time; $\mathrm{Cl}$, clearance; $V$, apparent volume of distribution. For the three kinds of emulsions, there were no significant differences between the BCOE and BJOE or CSOE $(p>0.05)$

Table 6. TGIR in $\mathrm{S}_{180}$ Sarcoma-Bearing Mice of the Different Medication Administration Teams

\begin{tabular}{|c|c|c|c|c|c|c|}
\hline Group & $\begin{array}{c}\text { Dose } \\
(\mathrm{ml} / \mathrm{kg} / \mathrm{d})\end{array}$ & $\begin{array}{l}\text { Animal numbers } \\
\text { begin/over }\end{array}$ & $\begin{array}{c}\text { Tumor weight }(\mathrm{g}) \\
\text { group I }\end{array}$ & $\begin{array}{c}\text { Tumor weight (g) } \\
\text { group II }\end{array}$ & $\begin{array}{c}\text { Tumor weight (g) } \\
\text { group III }\end{array}$ & TGIR (\%) \\
\hline Physiological saline & 10 & $12 / 12$ & $1.7371 \pm 0.4868$ & $1.5771 \pm 0.4297$ & $1.6263 \pm 0.3972$ & - \\
\hline Blank lipid emulsion & 10 & $12 / 12$ & $1.2845 \pm 0.4356$ & $1.6542 \pm 0.4027$ & $1.7409 \pm 0.4789$ & - \\
\hline CSOE i.v. & 10 & $12 / 12$ & $1.0729 \pm 0.4251$ & $1.1069 \pm 0.3917$ & $1.0357 \pm 0.4123$ & 34.8 \\
\hline BJOE i.v. & 10 & $12 / 12$ & $0.9875 \pm 0.3176$ & $0.9714 \pm 0.4261$ & $0.9801 \pm 0.3251$ & 40.4 \\
\hline BCOE-low i.v. & 5 & $12 / 12$ & $1.3297 \pm 0.3719$ & $1.2157 \pm 0.3495$ & $1.0753 \pm 0.3172$ & 26.7 \\
\hline BCOE -middle i.v. & 10 & $12 / 12$ & $0.9357 \pm 0.3517$ & $0.8794 \pm 0.3679$ & $0.8217 \pm 0.3963$ & 46.6 \\
\hline BCOE -high i.v. & 20 & $12 / 12$ & $0.8179 \pm 0.3127$ & $0.7319 \pm 0.2936$ & $0.7856 \pm 0.3002$ & 52.9 \\
\hline BCOE p.o. & 10 & $12 / 12$ & $1.4536 \pm 0.3297$ & $1.3876 \pm 0.3517$ & $1.4928 \pm 0.4617$ & 12.2 \\
\hline
\end{tabular}

The data are means \pm S.D., $n=12$. The experiments were carried out in triplication, which were described by group I, group II and group III.

Table 7. Determination of Mortality (the Dose Was $20 \mathrm{ml} / \mathrm{kg} / \mathrm{d}$, Administered via the Tail Vein) to Evaluate the Toxicity among BCOE, BJOE and CSOE

\begin{tabular}{ccccc}
\hline \hline Group & $\begin{array}{c}\text { Animal number at } \\
\text { beginning in each group }\end{array}$ & $\begin{array}{c}\text { Dead animals after } \\
\text { administration (I) }\end{array}$ & $\begin{array}{c}\text { Dead animals after } \\
\text { administration (II) }\end{array}$ & $\begin{array}{c}\text { Dead animals after } \\
\text { administration (III) }\end{array}$ \\
\hline Control & 10 & 1 & 0 & 0 \\
BJOE & 10 & 6 & 7 & 6 \\
BCOE & 10 & 0 & 0 & 0 \\
CSOE & 10 & 1 & 2 & 0 \\
\hline
\end{tabular}


role in maintaining the physical stability of the fat emulsion and ensuring the safety of intravenous application. ${ }^{18)}$ Also, the selection of emulsifiers has a direct influence on the emulsification process since different oils need to be emulsified with different emulsifiers. ${ }^{19)}$ It has been reported that only a limited number of emulsifiers are generally regarded as safe to use for parenteral administration, and the most important of these are F-68 and lecithin. Lecithin can be totally biodegraded and metabolized, because it is an integral part of biological membranes, making it virtually non-toxic. ${ }^{20)}$ In addition, due to its amphiphilic character and endogenous nature, lecithin is a popular raw material in the modern pharmaceutical industry, and it has been extensively used in preparations for parenteral nutrition intended for intravenous administration. ${ }^{21)}$ Among the many kinds of lecithin, Lipid E 80 is enriched in phosphatidylcholine (PC) and a few components in Lipid E 80 are negatively charged (phosphatidic acid (PA), phosphatidylserine (PS)) or uncharged at neutral $\mathrm{pH}$ values. $^{22)}$ Hansrani $^{23)}$ found that various minor lecithin components, like PA, phosphatidylinositol (PI), PS, are able to contribute to enhanced emulsion stability. Therefore, Lipid E 80 was selected as the main emulsifier in this paper. F-68 was also chosen as a co-emulsifier since it is rapidly dispersed at the oil-water film interface to increase the strength of the film and emulsify the oil phase. The results described in Table 1 show that the ratios in Formulations 3 to 5 result in the formation of a compact interfacial film producing an optimum emulsification. Considering that F-68 is not endogenous, the less used the better. Therefore, based on the satisfactory sterilization and storage stability, Formulation 3 with Lipid E 80:F-68 2:1 was taken as the most suitable ratio. The particle size in Table 1 also suggested that, with these three emulsifier ratios, BCOE is safe for intravenous administration and has sufficient stability to withstand thermal sterilization.

(b) The Total Amount of Lipid E 80 and F-68: Since the safety of intravenous emulsion is to a high degree due to the particle size distribution of the oil phase for particles larger than $5 \mu \mathrm{m}$ given intravenously can lead to emboli in vivo, ${ }^{24,25)}$ PSD was chosen as the estimating parameter. With the reduction in the total amount of Lipid E 80 and F-68, the particle size increased and the standard deviation decreased as shown in Table 2. This showed that the more emulsifiers used, the smaller the particle size obtained since the emulsification was more efficient. However, excess emulsifier, especially excess lecithin could increase the standard deviation because the excess lecithin can form small particles, such as phospholipid micelles and unilamellar liposomes. This phenomenon is obtained based on Fig. 1. It has been reported that excess emulsifier is the reason for the dislipidemia seen following long-term infusion. ${ }^{26)}$ Deviation from the chylomicronlike behaviour and, thus, a different metabolic fate of lecithin-coated oil droplets is presumed. This was again suspected of involving liposomes which caused enhanced formation of abnormal 'Lipoprotein-X', increased cholesterol release and other dislipidemic effects. ${ }^{27)}$ Hence, in the formulation promoted in this paper, $0.9 \%$ of Lipid E 80 and F-68 was selected, in order to obtain a satisfactory emulsification.

(c) The Amount of Sodium Oleate: The $\zeta$-potential reflects the surface charged properties of the particles, and is one of the most important parameters for evaluating the stability of dispersed systems. For emulsion systems, the $\zeta$-potential is usually maintained between $-20--45 \mathrm{mV}$. Sodium oleate is often used to adjust the $\zeta$-potential since it allows adsorption to the formed oil-water interfacial film, giving microparticles with a negative charge. ${ }^{28,29)}$ The negative charge on the emulsion surface causes electrical repulsion between micro-particles and, in addition, the electrical repulsion plays an important role in the physical stability of emulsions. It is obvious that the incorporation of sodium oleate makes an important contribution to the physical stability of the emulsion by increasing the absolute value of the $\zeta$-potential. Finally, $0.1 \%(\mathrm{w} / \mathrm{v})$ sodium oleate was used to prepare BCOE.

The formulation promoted in this paper finally consisted of $10 \%(\mathrm{w} / \mathrm{v}) \mathrm{BJO}$ and CSO 3:1, 0.6\% (w/v) lecithin, $0.3 \%$ $(\mathrm{w} / \mathrm{v}) \mathrm{F}-68,0.1 \%(\mathrm{w} / \mathrm{v})$ sodium oleate and $2.5 \%(\mathrm{w} / \mathrm{v})$ glycerin.

Preparation Investigation The properties of the final emulsion are largely dependent on the preparation techniques used. The emulsification temperature, homogenization cycles and pressure and sterilization procedure were systematically investigated in this study.

(a) $70^{\circ} \mathrm{C}$ Was Selected as the Temperature for Preparing Course Emulsion: $70^{\circ} \mathrm{C}$ is the phase inversion temperature of lecithin, ${ }^{30)}$ and preparation of emulsions close to this temperature could narrow the particle size distribution and, in addition, at $70^{\circ} \mathrm{C}$ the viscosity of the oils is low, therefore, the applied exterior shearing force needs less power, which benefits emulsification. So, $70^{\circ} \mathrm{C}$ was selected as the emulsification temperature when preparing a crude emulsion of BCOE. Moreover, Bock et al. ${ }^{31)}$ stated that the phase-inversion method led to a markedly shorter pre-emulsification step and, therefore, to reduced hydrolysis, which increased the emulsion stability. Hence, combining a water phase with an oil phase was selected to form a crude emulsion at $70^{\circ} \mathrm{C}$ in the study, and this also caused a phase-inversion between the aqueous and oil phases.

(b) Investigation of High Pressure Homogenization Cycles and Pressure ${ }^{32-35)}$ : Homogenization involves concurrent reduction of particle size and narrowing of the particle size distribution. ${ }^{36)}$ Intravenous emulsions must avoid capillary blockage and they also need to be more stable on creaming when droplets are uniformly small. Safety of use is to a high degree dependent on the particle size distribution of the oil phase, since particles larger than $5 \mu \mathrm{m}$ given intravenously can lead to emboli in vivo. ${ }^{24)}$ The stability of a parenteral emulsion is mainly used to maintain its main physical property, namely, a dispersed phase particle size distribution. The results in Table 3 indicated that more than 6 homogenization cycles had no significant effect on the emulsion particle size. Furthermore, the increasing of standard deviations following 10 homogenization cycles is probably because the excess pressure or prolonged homogenization led to overprocessing of the emulsions, when the droplet diameter and distribution increases, ${ }^{25}$ since emulsion particles undergo aggression and micelles or liposomes are formed. Moreover, It was reported that reprocessing of the emulsion during several homogenization cycles reduces the particle size distribution even further, while a major particle size reduction is already achieved after 2-3 cycles. ${ }^{33,34,37)}$ Hence, according to industrial production conditions, the homogenization pressure was set at 500 bar, and 6 homogenization cycles were chosen for the 


\section{BCOE preparation.}

(c) Investigation of Sterile Methods: It is suggested that a longer sterilization time might reduce the stability of the emulsion, and provide more opportunity for aggregation of emulsion particles. Therefore, according to the PSD and appearance shown in Table $4,115^{\circ} \mathrm{C}$ for $30 \mathrm{~min}$ was employed as thermal sterilization method.

The final procedure conditions involved $70^{\circ} \mathrm{C}$ for preparing a crude emulsion, 500 bar pressure, 6 cycles for homogenization, with the $\mathrm{pH}$ value was adjusted to 8.5 after high pressure homogenization and $115^{\circ} \mathrm{C}$ for $30 \mathrm{~min}$ in a rotating water vapor bath.

Pharmacokinetics in Rats after Intravenous Injection of BCOE Since oleic acid is the major component in BCOE and relatively little is present in blank plasma, and it is also the main ingredient in anti-tumor treatments, it was chosen as the component for determination. The method used for plasma had an acceptable recovery rate of $86.4 \%$ and was easy to carry out. The linear concentration range of oleic acid in plasma was $2.60-34.72 \mu \mathrm{g} / \mathrm{ml}$, and the interand intra-day variations were $5.5 \%$ and $9.6 \%$, respectively, and therefore both less than $15.0 \%$. Moreover, the active component oleic acid and internal standard heptadecanoic acid in BCOE were properly separated under the HPLC analytical conditions. The pharmacokinetics of oleic acid was evaluated by a noncompartmental method and the plasma concentration-time profiles and the parameters of the noncompartmental pharmacokinetic analysis between the BCOE and BJOE or CSOE were tested by an independent samplestest. The results obtained show that there were no significant differences between the BCOE and BJOE or CSOE. The elimination rate constant in each group suggested that all three emulsions were eliminated in vivo by a physical elimination process when a fixed amount of emulsifiers and a controlled range of particle sizes were used. Therefore, BJO and CSO had no effect on the elimination of BCOE.

Anti-tumor Studies of BCOE The anti-tumor activity and toxicity of BCOE were evaluated by determining the tumor growth inhibitory ratio (TGIR) and mortality. As shown in Table 6 , the $S_{180}$ tumor weight of the physiological saline control group and lipid emulsion control group were more than $1 \mathrm{~g}$, which proved that the tumor model was appropriate and furthermore, the adjuvant such as emulsifiers used in the BCOE were proved to have no intrinsic effect on tumors and the anti-tumor activity is produced by the intrinsic activity of BJO and CSO. Table 6 shows that the anti-tumor effect of CSOE was weaker than that of BJOE since the TGIR of the CSOE group, $34.8 \%$, was lower than that of the BJOE group, $40.4 \%$. Also, the result of the medium dosage group of $\mathrm{BCOE}$ was $46.6 \%$, which was higher than that of both the CSOE and BJOE groups. This shows that the combined use of the two oils produced a better anticancer therapeutic effect. It can also be presumed that the TGIR of BCOE was dose-dependent based on the TGIR obtained according to the BCOE group from the low dose to the high dose group, which were $26.7 \%, 46.6 \%$, and $52.9 \%$, respectively. The TGIR of BCOE for the p.o. group was only $12.2 \%$, which was much lower than that using intravenous administration. It suggested that the intravenous administration is markedly superior to oral administration in anticancer treatment. Moreover, the percentage mortality of BCOE shown in Table 7 was much lower than in the BJOE group, $63.3 \%$, and in CSOE, $13.3 \%$, which strongly support the belief that BCOE greatly reduced the toxicity of BJO and CSO. In a word, the anti-tumor activity studies demonstrate that BCOE maintains or even increases the anti-tumor activity as well as producing a clear reduction in toxicity compared with BJOE or CSOE.

In conclusion, the formulation and preparation of $\mathrm{BCOE}$ were thoroughly investigated in this study. The formulation promoted in this paper is composed of $10 \%(\mathrm{w} / \mathrm{v})$ oils with BJO and CSO 3:1, 0.6\% (w/v) Lipid E 80, 0.3\% (w/v) Pluronic F-68, 0.1\% (w/v) sodium oleate and 2.5\% (w/v) glycerin in water. Also, the final preparation conditions were $70{ }^{\circ} \mathrm{C}$ for preparing crude emulsions, 500 bar pressure, 6 cycles for homogenization, $\mathrm{pH}$ was adjusted to 8.5 after high pressure homogenization and $115^{\circ} \mathrm{C}$ for $30 \mathrm{~min}$ in a rotating water vapor bath as thermal sterilization conditions. PSD and $\zeta$-potential were used as the main parameters to evaluate the physical stability of emulsions. Based on the results of the pharmacokinetics and anti-tumor activity experiments, BCOE with compound oils enhances the anti-tumor activity and produces a better effect as well as a reduction in toxicity and adverse effects. Hence, the development of BCOE is very important and represents a marked advance in antitumor therapy.

Acknowledgment Dr. Yingliang $\mathrm{Wu}$ is gratefully thanked for the support during the anti-tumor activity studies.

\section{REFERENCES}

1) Alison G. F., J. PSTT, 2, 134-143 (1999).

2) Wretlind A., JPEN J. Parenter Enteral Nutr., 5, 230-235 (1981).

3) Dapeng L., J. CJIM., 10, 233-235 (2004).

4) Luyengi L., Suh N., Fong H. H., Pezzuto J. M., Kinghorn A. D., Phytochemistry, 43, 409-412 (1996).

5) Yuqing S., Guangen G., J. Shenyang Pharmaceutical University, 11 23-26 (1979).

6) Willinghan W. J., Stafford E. A., Reynolds S. H., Chaney S. G., Lee K. H., Okano M., Hall I. H., Biochim. Biophys. Acta, 654, 169-174 (1981).

7) Ying L., Gongli X., Ying L., Nan Zh., J. Clin. Hematol., 17, 154-156 (2004).

8) Bingsheng L., Wenlong R., Hongqi L., Xiuhua Ch., Yulong Y., Huifang Y., J. Chinese Pharmaceutical Industry, 299, 456-458 (1998).

9) Daming D., Jingping Zh., Chinese Pharmaceutical J., 35, 26-27 (2000).

10) Michael P., Harald K., Elke von O., Reinhard K., Clin. Chem., 39 825-832 (1993).

11) Saito M., Ushijima T., Sasamoto K., Ohkura Y., Ueno K., J. Chromatogr. B., 674, 167-175 (1995).

12) Wolf J. H., Korf J., J. Chromatogr., 502, 423-430 (1990).

13) Miwa H., Hiyama C., Yamamoto M., J. Chromatogr., 321, 165-174 (1985).

14) Miwa H., Yamamoto M., Nishida T., Nunoi K., Kikuchi M., J. Chromatogr., 416, 237-245 (1987).

15) Abushufa R., Reed P., Weinkove C., Clin. Chem., 40, 1707-1712 (1994).

16) Durst H. D., Milano M., Kikta E. J., Connelly S. A., Grushka E., Anal. Chem., 47, 1797-1801 (1975)

17) Jia Y., Haibing H., Xing T., J. Pharm. Pharmacol., 58, $1-7$ (2006).

18) Weingarten C., Magalhaes N. S. S., Baszkin A., Benoita S., Seiller M., Int. J. Pharm., 75, 171-179 (1991).

19) Muhannad J., Bernd W. M., Int. J. Pharm., 163, 81-89 (1998)

20) Rabinovich G. L., Dubernet C., Gaudin K., Lambert G., Couvreur P., 
Chaminade P., Eur. J. Pharm. Biopharm., 61, 69-76 (2005).

21) Huth K., Schoenborn W., Boerner J., Med. Ernaehr., 8, 146-148 (1967).

22) Szoka F. J., Papahadjopoulos D., Ann. Rev. Biophys. Bioeng., 9, 467508 (1980).

23) Hansrani P. K., Studies on intravenous fat emulsions. Ph.D. Thesis, University of Nottingham (1980).

24) Hadfield J. I. H., Clin. Med., 73, 25 (1966).

25) Davis S. S., J. Hosp. Pharm., 32, 149-170 (1974).

26) Lutz O., Meraihi Z., Ferezou J., Frey A., Lutton C., Bach A. C., Metabolism, 39, 1225-1231 (1990).

27) Bach A. C., Férézou J., Frey A., Prog. Lipid Res., 35, 133-153 (1996).

28) Muchtar S., Levy M. Y., Sarig S., Benita S., S. T. P. Pharma Sci., 2 $130-136$ (1991).

29) Yamaguchi T., Nishizaki K., Itai S., Hayashi H., Ohshima H., Pharm.
Res., 12, 342-347 (1995).

30) Tamilvanan S., Prog. Lipid Res., 43, 489-533 (2004).

31) Bock T. K., Lucks J. S., Kleinebudde P., Mueller R. H. Mueller B. W., Eur. J. Pharm. Biopharm., 40, 157-160 (1994).

32) Bock T., "Emulsionen als parenterale Arzneistofftraegersysteme-Herstellung, Charakterisierung und Optimierung," Ph.D. Thesis, Christian-Albrechts-University, Kiel, 1994.

33) Ishii F., Sasaki I., Ogata H., J. Pharm. Pharmacol., 42, 513-515 (1990).

34) Washington C., Davis S. S., Int. J. Pharm., 39, 33-37 (1987).

35) Washington C., Davis S. S., Int. J. Pharm., 44,169-176 (1988).

36) Walstra P., "Formation of Emulsions" "Encyclopedia of Emulsion Technology," Vol. 1, ed. by Becher P., Marcel Dekker Inc., New York, 1983.

37) Lidgate D. M., Fu R. C., Fleitman J. S., BioPharm., 45, 28-33 (1989). 